\title{
Revisiting the structure function of PSR B0950+08 scintillations
}

\author{
Itzhak Goldman ${ }^{1,2}$ * \\ ${ }^{1}$ Department of Physics, Afeka College, Tel Aviv, Israel \\ 2 Department of Astrophysics, Tel Aviv University, Tel Aviv, Israel
}

24 May 2022

\begin{abstract}
The observational structure function of the scintillations of the radio pulsar PSR B0950+08, was fitted, a decade ago, with a power law with index $1 \pm 0.01$. This was interpreted as an appreciable deviation from the, commonly observed index of $5 / 3$, expected for Kolmogorov turbulence.

In this paper it is suggested that the observations are consistent with a Kolmogorov turbulence and that the apparent deviation is due to a turbulent region with an effective depth which is comparable to the observed lateral scales on the plane of the sky, spanned by the pulsar beam. Alternatively, the fitted index of 1 is consistent with an underlying compressive turbulence and an even smaller depth.

In the first interpretation the depth is $(5.5 \pm 1.8) \times 10^{8} \mathrm{~cm}$. In the second one, the depth is $\lesssim 4 \times 10^{7} \mathrm{~cm}$. These estimates lend support for the existence of extremely thin, ionized scattering screens in the local interstellar cloud, that have been proposed a decade ago.
\end{abstract}

Key words: pulsars: general-pulsars: individual (PSR B0950+08)-turbulence-ism

\section{INTRODUCTION AND SUMMARY}

Pulsar scintillation proved to be an important tool for the study of the structure of the interstellar medium (ISM) (Howes (2008), Goodman \& Narayan (1985), Elmegreen \& Scalo (2004), Scalo \& Elmegreen (2004) and references therein ). The observational scintillations, generally imply structure functions that are power laws of the time lag between measurements.

Due to the pulsar tangential motion on the plan of the sky, relative to the turbulent region, the detected pulses scan different regions of the ISM in the course of time. Thus, the time lapse between successive detections, corresponds to an effective lateral distance in the turbulent region. This allows the observational temporal structure function to be interpreted in terms of spatial structure function of the electron density.

Analysis of observed scintillation data for many pulsars imply a spatial structure function of the spatial lateral scale $x$ typically, in the form $S(x) \propto x^{\alpha}$ with $\alpha \simeq 1.67$. This is consistent with the Kolmogorov spectrum (Kolmogorov (1941)) which characterizes subsonic homogeneous and isotropic turbulence. This power spectrum is quite common in various astrophysical settings (Elmegreen \& Scalo (2004), Scalo \& Elmegreen (2004) and references therein).

Observational structure functions which deviate from the Kolmogorov spectrum were also reported; see e.g. Stinebring et al. (2000). The largest such deviation was reported by Smirnova \& Shishov (2008), for the nearby pulsar PSRB0950+08. The fitted power law index to the derived

* E-mail:goldman@afeka.ac.il structure function was $1 \pm 0.05$. These authors concluded that it corresponds to a $3 \mathrm{D}$ power spectrum for the electron density having an index $-3 \pm 0.05$, considerably different from the Kolmogorov value $-11 / 3 \simeq-3.67$.

Here, we suggest two conservative alternative interpretations of the observational structure function. The first is consistent with the standard Kolmogorov turbulence and the second with compressive supersonic turbulence. Both interpretations imply that the turbulent region responsible for the scintillation has a very small depth.

The various scintillation observables are proportional to the column density of the electrons in the scattering region. The issue of power spectra of quantities which are the result of integration along the line-of-sight has been addressed by several authors (see e.g. Stutzki et al. (1998), Goldman (2000), Lazarian \& Pogosyan (2000), Miville-Deschênes, Levrier, \& Falgarone (2003)). They concluded that when the lateral spatial scale is much smaller than the depth of the layer, the logarithmic slope steepens exactly by -1 compared to its value when the lateral scale is much larger than the depth.

This behavior has been indeed observed in galactic and extragalactic turbulence (e.g. Elmegreen, Kim, \& StaveleySmith (2001), Miville-Deschênes et al. (2003), Block et al. (2010), Contini \& Goldman (2011)), and also in the power spectrum of the quiet Sun photospheric turbulence (Abramenko \& Yurchyshyn (2020) and Goldman (2020)).

Here, we show that also the observational structure function depends on the ratio between the spatial lags and the depth of the turbulent region. For a turbulence with a $1 \mathrm{D}$ power spectrum in the form of a power law with index $-m$, the observational structure function is a power law with in- 
dex $m-1$ for lateral lags much larger than the depth of the turbulent region. For lateral scales much smaller than this depth, the index is $m$.

For most pulsars, the depth is much larger than the observed spatial lags, resulting in an observed index $\sim 5 / 3$, in accordance with Kolmogorov spectrum. When the depth is much smaller than the lateral scale, the corresponding logarithmic slope is $\sim 2 / 3$.

The observational power spectrum of PSR B0950+08 is consistent with Kolmogorov turbulence if the lateral scales are comparable to the depth of the turbulent scattering screen. In this case the structure function will exhibit a transition between the two asymptotic limits. It can thus be mimicked by a single power law index $\sim 1$.

We note that an index of 1 is expected if the turbulence is supersonic with a $1 \mathrm{D}$ power which is a power law with index $m=2$ if the depth of the turbulence region is much smaller than the smallest lateral scale.

We obtain an estimate for the depth of the ionized region responsible for the fluctuations. The depth is rather small $(5.5 \pm 1.8) \times 10^{8} \mathrm{~cm}$, for Kolmogorov turbulence and $\lesssim 4 \times$ $10^{7} \mathrm{~cm}$ for compressive supersonic turbulence.

\section{THE 1-D TURBULENCE STRUCTURE FUNCTION OF LINE-OF-SIGHT INTEGRATED DATA}

Consider an observational fluctuating quantity $f(t)$ (time shifts, phase shifts or intensity). Its observational structure functions is defined in terms of an ensemble average (angular brackets) which (based on the ergodic principle) is evaluated as a time average.

A plane parallel geometry is assumed, in line with the small angular scales covered during the time lags $t . f(t)$ is proportional to the electron column density

$f(t, D) \propto \int_{0}^{D} n_{e}(t, z) d z$

where $D$ denotes the effective depth of ionized turbulent region causing the scintillations. It is termed "effective" because it is tacitly assumed in equation (1) that the depth does not depend on the lateral position.

The observational structure function is therefore

$S_{f}(t, D) \propto \int_{0}^{D} \int_{0}^{D}\left\langle\left(n_{e}\left(t+t^{\prime}, z\right)-n_{e}\left(t^{\prime}, z^{\prime}\right)\right)^{2}\right\rangle d z d z^{\prime}$

The structure function can be expressed as a function of lateral spatial lags $x$, related to the time lag $t$ due to a relative tangential velocity, $v$ between the scattering region and the line of sight: $x=v t$.

Thus, the observational structure function $S_{f}(t, D)$, can be regarded as a function $S_{f}(x, D)$. In this representation, it probes the turbulence in the ionized scattering screen. By definition

$$
\begin{aligned}
S_{f}(x, D) & \propto \int_{0}^{D} \int_{0}^{D}\left\langle\left(n_{e}\left(x+x^{\prime}, z\right)-n_{e}\left(x^{\prime}, z^{\prime}\right)\right)^{2}\right\rangle d z d z^{\prime} \\
& \propto \int_{0}^{D} \int_{0}^{D} S_{n_{e}}\left(x, z-z^{\prime}\right) d z d z^{\prime}
\end{aligned}
$$

where $S_{n_{e}}\left(x, z-z^{\prime}\right)$ is the structure function of the electron density fluctuations. Isotropy has been assumed imply- ing that the electron structure function depends on the relative spatial separation.

The electron fluctuations structure function is expressed by the electron density correlation function, as

$$
S_{n_{e}}\left(x, z-z^{\prime}\right)=2\left(C_{n_{e}}(0,0)-C_{n_{e}}\left(x, z-z^{\prime}\right)\right)
$$

with the correlation function

$$
\begin{array}{r}
C_{n_{e}}\left(x, z-z^{\prime}\right)=\left\langle n_{e}\left(x+x^{\prime}, z\right) n_{e}\left(x^{\prime}, z^{\prime}\right)\right\rangle= \\
\int_{-\infty}^{\infty} \int_{-\infty}^{\infty} e^{i\left(k_{x} x+k_{z}\left(z-z^{\prime}\right)\right)} P_{2}\left(k_{x}, k_{z}\right) d k_{x} d k_{z}
\end{array}
$$

where $P_{2}\left(k_{x} \cdot k_{y}\right)$ is the 2 -dimensional power spectrum. Therefore, the $1 \mathrm{D}$ correlation is

$$
\begin{array}{r}
C_{f}(x, D)=\int_{0}^{D} \int_{0}^{D} C_{n_{e}}\left(x, z-z^{\prime}\right) d z z^{\prime} \\
\propto \int_{-\infty}^{\infty} \int_{-\infty}^{\infty} e^{i k_{x} x} \sin ^{2}\left(k_{z} D / 2\right)\left(k_{z} D / 2\right)^{-2} d k_{x} d k_{z}
\end{array}
$$

Using equations(3),(4) one gets

$$
\begin{array}{r}
S_{f}(x, D) \propto \int_{0}^{\infty} \int_{0}^{\infty} \sin ^{2}\left(k_{x} x / 2\right) \sin ^{2}\left(k_{z} D / 2\right)\left(k_{z} D / 2\right)^{-2} \\
P_{2}\left(k_{x}, k_{z}\right) d k_{x} d k_{z}
\end{array}
$$

For a turbulence with a $1 \mathrm{D}$ spectrum which is a power law with index $-m$, the $2 \mathrm{D}$ power spectrum is

$P_{2}\left(k_{x}, k_{y}\right) \propto\left(k_{x}^{2}+k_{z}^{2}\right)^{-(m+1) / 2} ;$

so that

$$
\begin{gathered}
S_{f}(x, D)=M \int_{0}^{\infty} \int_{0}^{\infty}\left(k_{x}^{2}+k_{z}^{2}\right)^{-(m+1) / 2} \\
\sin ^{2}\left(k_{z} D / 2\right)\left(k_{z} D / 2\right)^{-2} \sin ^{2}\left(k_{x} x / 2\right) d k_{z} d k_{x}
\end{gathered}
$$

where $M$ is a constant.

Introducing the dimensionless variables

$\eta=k_{z} D / 2 \quad ; \quad \mu=k_{x} D / 2$

One can express the structure function of equation (10) in the form

$S_{f}(x, D)=N \int_{0}^{\infty} I(\mu) \sin ^{2}(\mu x / D) d \mu$

where $N$ is a constant and $I(\mu)$ is

$I(\mu)=\int_{0}^{\infty}\left(\mu^{2}+\eta^{2}\right)^{-(m+1) / 2} \frac{\sin ^{2} \eta}{\eta^{2}} d \eta$

Equation(11) implies that the structure function argument is $x / D$. Also, inspection of this equation reveals that for $x<<$ $D$ it is proportional to $x^{m}$ while for $x>>D$ it is proportional to $x^{m-1}$. The transition ratio $x_{t} / D$ can be estimated as the tangent point of a power law proportional to $x^{(m-0.5)}$. This ratio depends on the value of $m$.

Fig.1 displays the structure function for Kolmogorov turbulence $(m=5 / 3)$ in arbitrary units, as a function of the dimensionless ratio $x / D$. One clearly sees the asymptotic behaviors with logarithmic slopes of $2 / 3$ and $5 / 3$. The transition 


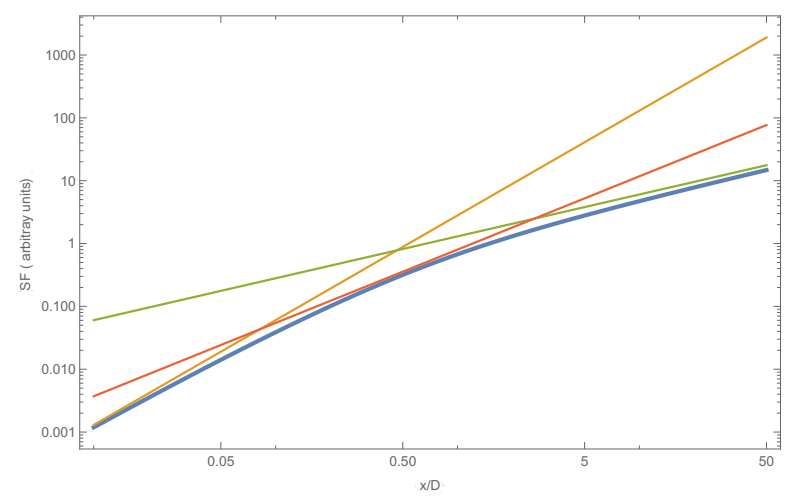

Figure 1. Blue line:The structure function for a Kolmogorov turbulence, in arbitrary units, as function of $x / D$. Shown also two asymptotes to the structure function. Orange line: limit $x<<D$ the power law index is $5 / 3$. Green line:in the limit $x>>D$ the power law index is $2 / 3$. Red line: a tangent to the structure function with logarithmic slope of $7 / 6$, used to define the transition lag.

value $x_{t} / D$ is defines as the tangent point of a power law logarithmic slope of $7 / 6$. The value obtained is $x_{t} / D=0.55$ so that

$D=1.82 x_{t}$

where $x_{t}$ is the transition scale in the observational structure function.

\section{APPLICATION TO THE OBSERVATIONAL STRUCTURE FUNCTION OF PSR B0950+08}

Let us apply the results of the previous section to the observational structure function of PSR B0950+08. Fig. 2 displays the observational structure function of PSR B0950+08 adopted from Fig. 6 of Shishov and Smirnova (2008). The spatial lateral lag $x$ was obtained with $v=36.6 \mathrm{~km} / \mathrm{s}$ employed there. The blue thick curve is the best fit of the structure function of Fig. 1 to the data. From the fit we obtain $x_{t}=(3 \pm 1) \times 10^{8} \mathrm{~cm}$. Therefore, using equation (12) one gets

$D=(5.5 \pm 1.8) \times 10^{8} \mathrm{~cm}$

Finally, we display in Fig. 3 the original fit to a power law with index 1 and the present fit. On the basis of the goodness of the fit, measured by least squares method, the two are on the same level. In the next section, we suggest that the fit to a power law with index 1 can be ascribed to a compressive turbulence with $1 \mathrm{D}$ power spectrum for which $m=2$.

\section{DISCUSSION}

We analyzed the structure function of an observable which is the result of integration along the line of sight. It has been shown that for a $3 \mathrm{D}$ turbulence spectrum which is a power low of the wavenumber with index $-(m+2)$ the 1D structure function exhibits two asymptotic behaviors. For spatial lags much larger than the depth of the turbulent layer it is a power law with index $m-1$, while for lags much smaller than the depth, the index is $\mathrm{m}$. Thus, an observed power

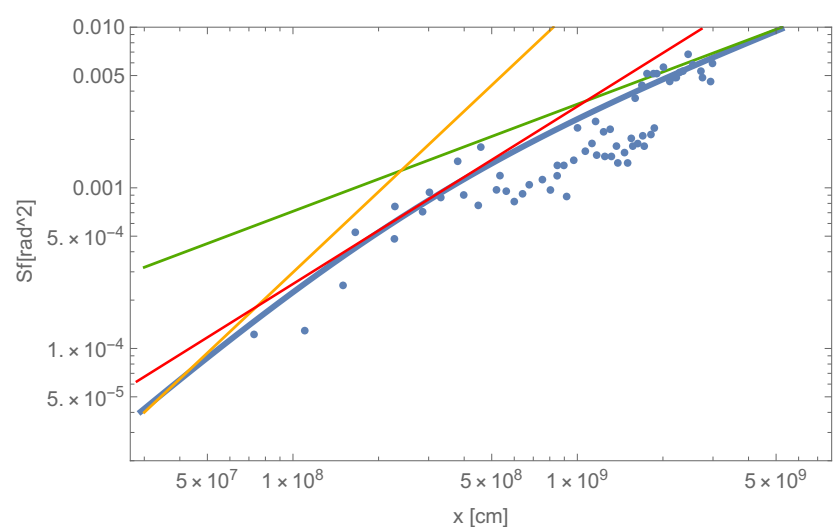

Figure 2. The best fit of the structure function to the observational values. The asymtotes are shown. From the graph one obtains $x_{t}=$ $(3 \pm 1) \times 10^{8} \mathrm{~cm}$, implying $\left.D=5.5 \pm 1.8\right) \times 10^{8} \mathrm{~cm}$.

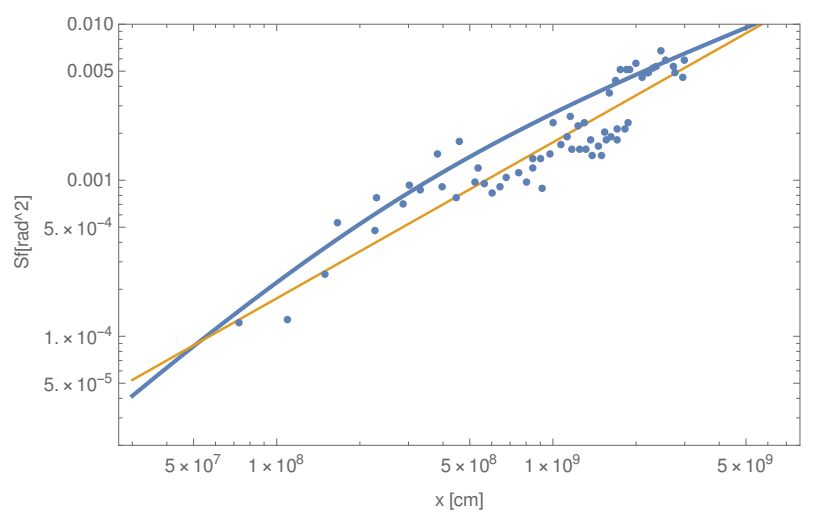

Figure 3. it Dots: observational values from Shishov and Smirnova (2008). Blue curve: the best fit spectrum - a depth of $5.5 \times 10^{8} \mathrm{~cm}$. Orange line: a power law with index 1

law with index $\sim 5 / 3$ is indicative of Kolmogorov turbulence case where the largest spatial lags are smaller than the depth. This is indeed, the common situation. Nevertheless, a two slope structure function was actually observed for PSR06531 (Stinebring et al. 2000) but was not interpreted in the way proposed here.

For the specific case of PSR B0950+08, the observations are consistent with the Kolmogorov spectrum provided that the spatial lags and the depth are comparable in size. Specifically in the present case the depth was estimated to be $D=(5.5 \pm$ $1.8) \times 10^{8} \mathrm{~cm}\left(v / 36.6 \mathrm{kms}^{-1}\right)$ where $v$ denotes the relative tangential velocity between the line of sight and the turbulent region.

Alternatively, a logarithmic slope of 1 can fit a compressive supersonic turbulence for which $m=2$, if the smallest observational spatial lags are much larger than the depth of the turbulent layer. In this case, an even smaller depth is implied: $D \lesssim 4 \times 10^{7} \mathrm{~cm}$.

Compressive supersonic turbulence characterized by a $1 \mathrm{D}$ power spectrum with index $m=2$ has been observed in molecular clouds (e.g. Larson (1981), Dame et al. (1986), Falgarone, Puget, \& Perault (1992)), in HII spectra of star forming regions (Roy \& Joncas (1985)) in HI emission ( Green (1993), Stanimirovic et al. (1999)) in a shocked nebula near the Galactic center (Contini \& Goldman 2011) and in 
numerical simulations (e. g. Passot, Pouquet, \& Woodward (1988),Vázquez-Semadeni, Ballesteros-Paredes, \& Rodríguez (1997)).

It is relevant to note here, that Linsky, Rickett, \& Redfield (2008) and Redfield \& Linsky (2008) observed in the local interstellar cloud (LIC) thin scattering screens at edges of adjacent clouds with relative supersonic velocities. Forming shocks can indeed create dense thin filaments with compressive supesonic turbulence. Thus, this interpretation seems plausible.

\section{ACKNOWLEDGMENTS}

I thank the Afeka College Research Authority for support.

\section{DATA AVAILABILITY}

This is a theoretical paper. The sole observational data used are from the paper of Smirnova \& Shishov (2008).

\section{REFERENCES}

Abramenko V. I., Yurchyshyn V. B., 2020, MNRAS, 497, 5405

Block D. L., Puerari I., Elmegreen B. G., Bournaud F., 2010, ApJ, $718, \mathrm{~L} 1$

Contini M., Goldman I., 2011, MNRAS, 411, 792

Dame T. M., Elmegreen B. G., Cohen R. S., Thaddeus P., 1986, ApJ, 305, 892

Dennett-Thorpe J., de Bruyn A. G., 2001, Ap\&SS, 278, 101

Elmegreen B. G., Kim S., Staveley-Smith L., 2001, ApJ, 548, 749

Elmegreen B. G., Scalo J., 2004, ARA\&A, 42, 211

Falgarone E., Puget J.-L., Perault M., 1992, A\&A, 257, 715

Goldman I., 2020, MNRAS, 499, 5363

Goldman I., 2000, ApJ, 541, 701

Goodman J., Narayan R., 1985, MNRAS, 214, 519

Green D. A., 1993, MNRAS, 262, 327.

Howes G. G., 2008, PhPl, 15, 055904

Kolmogorov A., 1941, DoSSR, 30, 301

Larson R. B., 1981, MNRAS, 194, 809

Lazarian A., Pogosyan D., 2000, ApJ, 537, 720

Linsky J. L., Rickett B. J., Redfield S., 2008, ApJ, 675, 413

Miville-Deschênes M.-A., Levrier F., Falgarone E., 2003, ApJ, 593, 831

Miville-Deschênes M.-A., Joncas G., Falgarone E., Boulanger F., 2003, A\&A, 411, 109

Passot T., Pouquet A., Woodward P., 1988, A\&A, 197, 228

Redfield S., Linsky J. D., 2008, ApJ, 673, 283

Roy J.-R., Joncas G., 1985, ApJ, 288, 142

Scalo J., Elmegreen B. G., 2004, ARA\&A, 42, 275

Smirnova T. V., Shishov V. I., 2008, ARep, 52, 736

Stanimirovic S., Staveley-Smith L., Dickey J. M., Sault R. J., Snowden S. L., 1999, MNRAS, 302, 417

Stinebring D. R., Smirnova T. V., Hankins T. H., Hovis J. S., Kaspi V. M., Kempner J. C., Myers E., Nice D. J., 2000, ApJ, 539, 300

Stutzki J., Bensch F., Heithausen A., Ossenkopf V., Zielinsky M., 1998, A\&A, 336, 697

Vázquez-Semadeni E., Ballesteros-Paredes J., Rodríguez L. F., 1997, ApJ, 474, 292 